\title{
Pharmacokinetic modeling of Gadolinium nanoparticles (Gd-NPs) with the sojourn time in vasa vasorum for the contrast enhanced MRI
}

\author{
Angkhana Prommarat ${ }^{1,2}$ and Farida Chamchod ${ }^{1,2^{*}}$
}

\author{
"Correspondence: \\ farida.cha@mahidol.ac.th \\ 'Department of Mathematics, \\ Faculty of Science, Mahidol \\ University, 10400 Bangkok, Thailand \\ ${ }^{2}$ Center of Excellence in \\ Mathematics, CHE, 10400 Bangkok, \\ Thailand
}

\begin{abstract}
Deposition of lipid in the artery wall called atherosclerosis is recognized as a major cause of cardiovascular disease that leads to death worldwide. A better understanding into factors that may influence the delivery of gadolinium nanoparticles (Gd-NPs) that enhances quality of magnetic resonance imaging in diagnosis may provide a vital key for atherosclerotic treatment. In this study, we propose a delay differential model for describing the dynamics of Gd-NPs in bloodstream, peripheral arteries, and vasa vasorum with two phenomena of Gd-NPs during a sojourn in vasa vasorum. We then investigate the dynamical behaviors of Gd-NPs and explore the effects of sojourn time and transfer rates of Gd-NPs on the concentration of Gd-NPs in vasa vasorum at the 12th hour after the administration of gadolinium chelates contrast media and also the maximum concentration of Gd-NPs in peripheral arteries and vasa vasorum. Our results suggest that the sojourn of Gd-NPs in vasa vasorum may lead to complex behaviors of Gd-NPs dynamics, and transfer rates of Gd-NPs may have a significant impact on the concentration of Gd-NPs.
\end{abstract}

Keywords: Atherosclerosis; Enhancement of MRl; Particle dynamics; Delay differential equations; Periodic oscillation

\section{Introduction}

Atherosclerosis, which is a common cardiovascular disease involving inflammation and accumulation of lipid plaques within the arterial wall, is recognized as a major cause of death worldwide $[1,2]$. There is recent evidence suggesting that vasa vasorum, which is a network of microvessels contained in tunicae adventitia that supply oxygen and nutrients to the arterial wall, is associated with the occurrence of atherosclerotic plaque progression $[3,4]$. For normal people, the oxygen is usually delivered by vasa vasorum and lumen to respectively reach outer and inner layers of the arterial wall. However, for atherosclerotic patients, vasa vasorum has changed in shape due to angiogenic expansion resulting in it becoming an only supply source for the entire wall $[5,6]$. Consequently, observation of vasa vasorum expansion can clinically help predict the pathogenesis of atherosclerosis [7].

(c) The Author(s) 2020. This article is licensed under a Creative Commons Attribution 4.0 International License, which permits use sharing, adaptation, distribution and reproduction in any medium or format, as long as you give appropriate credit to the original author(s) and the source, provide a link to the Creative Commons licence, and indicate if changes were made. The images or other third party material in this article are included in the article's Creative Commons licence, unless indicated otherwise in a credit line to the material. If material is not included in the article's Creative Commons licence and your intended use is not permitted by statutory regulation or exceeds the permitted use, you will need to obtain permission directly from the copyright holder. To view a copy of this licence, visit http://creativecommons.org/licenses/by/4.0/. 
Magnetic resonance imaging (MRI) is an important technique currently used to diagnose and detect the pathogenesis of early plaque. It is known that contrast agents are clinically employed to help enhance the quality of medical imaging modalities $[8,9]$. Usually, the contrast agents are presented in forms of nanoparticles (NPs) or liposomes containing metal ions such as iron(II), iron(III), gadolinium(II), gadolinium(III), manganese(II), or manganese(III) [10-12]. Gadolinium(III) complexes (gadolinium-based contrast agents (GBCAs) or gadolinium nanoparticles (Gd-NPs)) are the most widely clinically used agent through the intravenous route [13]. The working principle of Gd-NPs to assist the image acquisition is binding between the Gd-NPs and the specific biological environment such as molecular markers of disease. For atherosclerosis, there are $\alpha_{\nu} \beta_{3}$-integrins acting as the receptors of inflammatory endothelial cells on vasa vasorum, and Gd-NPs are designed and synthesized in a variety of chemical methods with the aim of targeting the $\alpha_{\nu} \beta_{3}$-integrin receptors $[6,14,15]$.

Even though the integrin binding helps enhance MR images, there are concerns about the safety of the contrast agents. In order to investigate factors that may influence their delivery and to predict possible effects inside the organs, physiologically-based pharmacokinetic (PBPK) models have often been chosen as important tools in many research studies. To investigate the multi-modality imaging technologies, Bartlett et al. [16] developed a three-compartmental model for plasma, interstitial and intracellular fluids to investigate the biodistribution of transferrin (tf)-targeted and nontargeted siRNA nanoparticles and the cellular uptake of a tumor. The study indicated that the tf-targeted NPs were more effective in attaching to the cell target and can help reduce tumor activity by 50 percent. Barboriak et al. [17] constructed three PBPK models including well-mixed, delay, and dispersion models to study the concentration-time curves (CTCs) of the gadolinium contrast agent at three position organs, which are cerebral cortex, superior sagittal sinus, and psoas muscle. Their results suggest that the best model to predict Gd-CTCs is the dispersion model. Neubauer et al. [18] examined the behavior of perfluorocarbon NPs while they were transported in blood, peripheral, and vasa vasorum by using a three-compartmental pharmacokinetic model. In their work, the NPs have the ability to target $\alpha_{v} \beta_{3}$-integrin receptors of atherosclerotic tissue resulting in the enhancement of contrast MR images. The purpose of their study was to develop models that are compatible with the new drug carriers. Wenger et al. [19] proposed a mathematical model based on a mass balance equation to evaluate distribution, accumulation, and excretion of the synthesized two NPs, polyacrylamide (PAA) and polyethylene glycol-coated polyacrylamide (PAApeg). Taheri et al. [20] studied the pharmacokinetics of Gd-DTPA administered at a bolus dose to optimize an area under the curve (AUC) of Gd-DTPA and found that reduction of Gd-DTPA together with optimized acquisition of DCE-MRI results in shorter acquisition time and less exposure of subjects.

In this present study, we incorporate the time-delay terms that reflect the sojourn of GdNPs in vasa vasorum into the mathematical model developed [18]. We also explore two types of functions describing circulation of Gd-NPs during the temporary stay in vasa vasorum: with and without natural clearance of Gd-NPs. Our goals are to (i) investigate the dynamical behaviors of Gd-NPs including periodic, asymptomatic, and oscillating solutions in bloodstream, peripheral arteries, and vasa vasorum; and (ii) explore the effects of certain parameters such as the sojourn time that Gd-NPs stay in vasa vasorum and transfer rates between the compartments on the concentration of Gd-NPs in vasa vasorum at 
the 12th hour after administration and the maximum concentration of Gd-NPs within peripheral arteries and vasa vasorum.

\section{Pharmacokinetic model}

Previously, a three-compartmental pharmacokinetic model was proposed to investigate the dynamical behaviors of Gd-NPs involved in the enhancement of MRI of early atherosclerosis [18]. Those three compartments represent bloodstream $\left(y_{1}\right)$, peripheral arteries $\left(y_{2}\right)$, and vasa vasorum $\left(y_{3}\right)$. In this work, the previous model is further extended to incorporate a fixed length of the sojourn time $(\tau)$ that Gd-NPs remain trapped in the third compartment so that the MR image signal remains elevated in comparison to the blood signal. Transfer of Gd-NPs between the first two compartments occurs at rates $k_{12}$ and $k_{21}$. Transfer of Gd-NPs between the third compartment and the first compartment occurs at rates $k_{13}$ and $k_{31}$. In addition to the distribution phase of Gd-NPs, the model also includes the clearance phase since Gd-NPs are normally removed from the bloodstream by phagocytosis in the reticuloendothelial system. An elimination constant for the clearance phase is denoted by $k_{e}$. As specific binding of $\alpha_{v} \beta_{3}$-targeted Gd-NPs to $\alpha_{v} \beta_{3}$-integrin can take place in vasa vasorum to enhance the MR signal, such transfer may include both passive and active transports of Gd-NPs. Consequently, the dynamics of Gd-NPs in those three compartments can be described by

$$
\begin{aligned}
& \frac{d y_{1}(t)}{d t}=-\left(k_{12}+k_{13}+k_{e}\right) y_{1}(t)+k_{21} y_{2}(t)+k_{31} y_{3}(t-\tau) f(\tau), \\
& \frac{d y_{2}(t)}{d t}=k_{12} y_{1}(t)-k_{21} y_{2}(t), \\
& \frac{d y_{3}(t)}{d t}=k_{13} y_{1}(t)-k_{31} y_{3}(t-\tau) f(\tau),
\end{aligned}
$$

where $y_{1}(t), y_{2}(t)$, and $y_{3}(t)$ represent the concentration of Gd-NPs at time $t(\mu M)$ in bloodstream, peripheral arteries, and vasa vasorum, respectively. In the model, $f$ is a function of $\tau$ where $\tau$ represents the sojourn time in the vasa vasorum compartment. Two types of $f$ are considered here to describe two different behaviors of Gd-NPs. The first case is $f(\tau)=1$, which reflects the completely retained amount of Gd-NPs during their temporary stay in the vasa vasorum compartment. The second case is $f(\tau)=e^{-k_{31} \tau}$, which reflects the decreasing amount of Gd-NPs at rate $k_{31}$ during the sojourn stay in the vasa vasorum compartment. To tackle the delay system of equations in (1) numerically, the corresponding non-delayed system is initially solved with the initial conditions

$$
y_{1}(0)=\frac{D}{v_{1}}, \quad y_{2}(0)=0, \quad y_{3}(0)=0
$$

on the time interval $t \in[0, \tau]$, where $D$ is the gadolinium dosage of $4.6 \mu \mathrm{mol} \mathrm{Gd} / \mathrm{kg}$ body weight injected to each test subject and $v_{1}$ is the distribution volume of bloodstream. Such a model is solved until the time reaches $t=\tau$ to obtain a new set of initial conditions for (1) on $[0, \tau]$ as follows:

$$
y_{1}(\theta)=Y_{1}^{0}(\theta), \quad y_{2}(\theta)=Y_{2}^{0}(\theta), \quad y_{3}(\theta)=Y_{3}^{0}(\theta), \quad \theta \in[0, \tau],
$$

where $Y_{i}^{0}(\theta)$ for $i=1,2,3$ denotes a solution of the corresponding non-delayed system at time $\theta$. For $t \in(\tau, \infty)$, the delay system is solved with conditions in (2). Parameters used in 
Table 1 Model parameters [18]

\begin{tabular}{llll}
\hline Name & Description & Targeted & Unit \\
\hline$k_{12}$ & Transfer rate from peripheral arteries to bloodstream & 1.220 & $1 / \mathrm{h}$ \\
$k_{21}$ & Transfer rate from bloodstream to peripheral arteries & 1.290 & $1 / \mathrm{h}$ \\
$k_{13}$ & Transfer rate from bloodstream to vasa vasorum & 1.100 & $1 / \mathrm{h}$ \\
$k_{31}$ & Transfer rate from vasa vasorum to bloodstream & 1.400 & $1 / \mathrm{h}$ \\
$k_{e}$ & Clearance rate from bloodstream & 0.126 & $1 / \mathrm{h}$ \\
$v_{1}$ & Volume of blood & 0.061 & $\mathrm{~L} / \mathrm{kg}$ \\
\hline
\end{tabular}

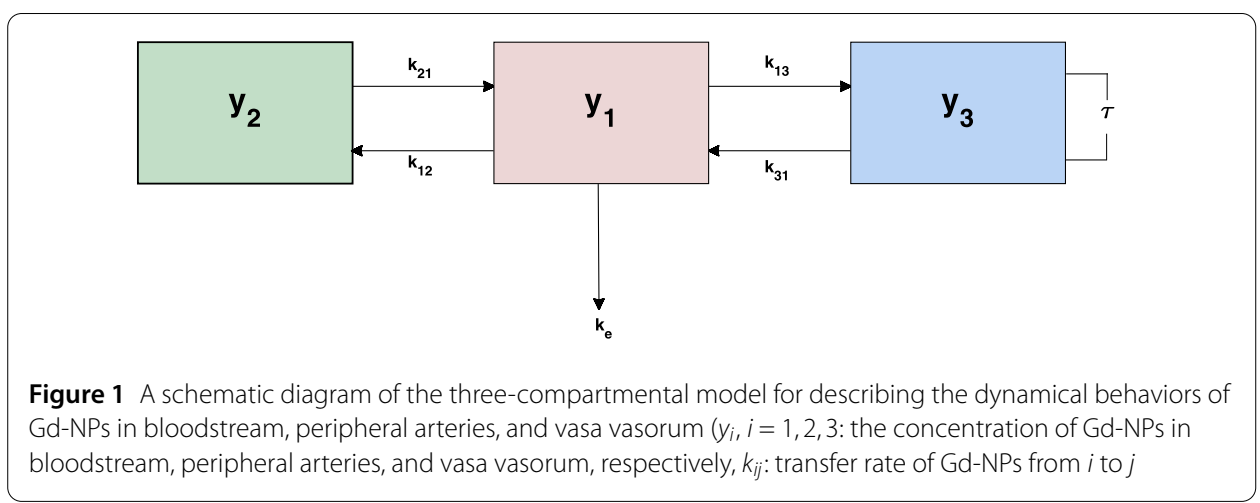

the model are presented in Table 1, while the flow diagram for describing the dynamical behaviors of Gd-NPs is illustrated in Fig. 1.

Note that model (1) can be extended to incorporate diffusion and chemotaxis of GdNPs. Examples of preceding studies that take into account those terms are such as Li et al. [21] and Viglialoro and Woolley [22].

\section{Analytic results}

Some analytic results of the model including an equilibrium and certain stability conditions are demonstrated in this section. Some other works related to the analysis of oscillatory and periodic solutions in detail can be found in [23] and [24]. By setting each equation in (1) equaling zero, the Gd-NPs-free equilibrium is obtained, $E^{0}=\left(y_{1}^{0}, y_{2}^{0}, y_{3}^{0}\right)=(0,0,0)$.

3.1 $\tau=0$ and $f(\tau)=1$

Theorem 1 The Gd-NPs-free equilibrium is locally asymptotically stable.

Proof First, we compute the eigenvalues $(\lambda)$ of the Jacobian matrix

$$
J=\left[\begin{array}{ccc}
-\left(k_{12}+k_{13}+k_{e}\right) & k_{21} & k_{31} \\
k_{12} & -k_{21} & 0 \\
k_{13} & 0 & -k_{31}
\end{array}\right]
$$

from the equation, $\operatorname{det}(J-\lambda I)=0$. The characteristic equation of $J$ is given by

$$
P(\lambda)=\lambda^{3}+a_{1} \lambda^{2}+a_{2} \lambda+a_{3}=0
$$

with

$$
a_{1}=k_{21}+k_{12}+k_{13}+k_{e}+k_{31}
$$




$$
\begin{aligned}
& a_{2}=k_{21} k_{13}+k_{21} k_{e}+k_{21} k_{31}+k_{31} k_{12}+k_{31} k_{e}, \\
& a_{3}=k_{21} k_{31} k_{e} .
\end{aligned}
$$

It is clear that $a_{1}>0, a_{3}>0$, and $a_{1} a_{2}>a_{3}$. Hence, according to the Routh-Hurwitz criteria [25], all eigenvalues of $J$ have negative real parts. Consequently, $E^{0}$ is locally asymptotically stable.

\section{$3.2 \tau \neq 0$ and $f(\tau)=1$}

For this case, as an equilibrium solution does not depend on time, there exists the GdNPs-free equilibrium of (1), $E^{0}$. To investigate the stability of $E^{0}$, let us define

$$
x(t)=y_{1}(t), \quad y(t)=y_{2}(t), \quad z(t)=y_{3}(t) .
$$

Then the linearized system of (1) in a matrix form can be described by

$$
\frac{d}{d t}\left(\begin{array}{l}
x(t) \\
y(t) \\
z(t)
\end{array}\right)=A_{1}\left(\begin{array}{l}
x(t) \\
y(t) \\
z(t)
\end{array}\right)+A_{2}\left(\begin{array}{l}
x(t-\tau) \\
y(t-\tau) \\
z(t-\tau)
\end{array}\right)
$$

where $A_{1}$ and $A_{2}$ are given by

$$
A_{1}=\left(\begin{array}{ccc}
-\left(k_{12}+k_{13}+k_{e}\right) & k_{21} & 0 \\
k_{12} & -k_{21} & 0 \\
k_{13} & 0 & 0
\end{array}\right), \quad A_{2}=\left(\begin{array}{ccc}
0 & 0 & k_{31} f(\tau) \\
0 & 0 & 0 \\
0 & 0 & -k_{31} f(\tau)
\end{array}\right)
$$

The characteristic equation of the linearized system is given by

$$
\triangle(\lambda)=\left|\lambda I-A_{1}-e^{-\lambda \tau} A_{2}\right|=0 .
$$

Hence,

$$
Q(\lambda, \tau)=\lambda^{3}+b_{1} \lambda^{2}+b_{2} \lambda+\left(b_{3}+b_{4} \lambda+b_{5} \lambda^{2}\right) e^{-\lambda \tau}=0
$$

with

$$
\begin{aligned}
& b_{1}=k_{12}+k_{21}+k_{13}+k_{e}, \\
& b_{2}=\left(k_{13}+k_{e}\right) k_{21}, \\
& b_{3}=k_{21} k_{31} k_{e}, \\
& b_{4}=\left(k_{12}+k_{21}+k_{e}\right) k_{31}, \\
& b_{5}=k_{31} .
\end{aligned}
$$

Since $\tau$ is transcendental and the system has infinitely many eigenvalues, investigating the stability of $E^{0}$ is much more difficult. Hence, we shall instead explore the distribution of roots of Eq. (3). 
By Rouche's theorem and the continuity in $\tau$, Eq. (3) has positive real parts if and only if it has purely imaginary roots. Then we study whether we can find purely imaginary roots. Assume that $\lambda(\tau)=i \omega(\tau)$ with $\omega>0$. Substituting $\lambda(\tau)$ into Eq. (3) gives

$$
\left(-i \omega^{3}-b_{1} \omega^{2}+i b_{2} \omega\right)+\left(b_{3}-b_{5} \omega^{2}+i b_{4} \omega\right)(\cos \omega \tau-i \sin \omega \tau)=0
$$

By separating real and imaginary parts and squaring their equations, we obtain

$$
\omega^{6}+\left(b_{1}^{2}-2 b_{2}-b_{5}^{2}\right) \omega^{4}+\left(b_{2}^{2}+2 b_{3} b_{5}-b_{4}^{2}\right) \omega^{2}-b_{3}^{2}=0
$$

Next, let

$$
Z=\omega^{2}, \quad \alpha=b_{1}^{2}-2 b_{2}-b_{5}^{2}, \quad \beta=b_{2}^{2}+2 b_{3} b_{5}-b_{4}^{2}, \quad \gamma=-b_{3}^{2} .
$$

Consequently, Eq. (5) can be rewritten as

$$
h(Z)=Z^{3}+\alpha Z^{2}+\beta Z+\gamma=0
$$

Proposition 1 Because $\gamma<0$, Eq. (6) has at least one positive root, denoted by $Z_{0}$.

Proof If $Z=0$, it follows that $h(0)=\gamma$. Since $h(0)<0$ and $\lim _{x \rightarrow \infty} Z^{3}+\alpha Z^{2}+\beta Z+\gamma=\infty$, Eq. (6) has at least one positive root. Consequently, let $\lambda(\tau)=\mu(\tau)+i \omega(\tau)$ be the eigenvalue of Eq. (3) such that $\mu\left(\tau_{0}\right)=0$ and $\omega\left(\tau_{0}\right)=\omega_{0}$. According to the separated real and imaginary parts of Eq. (4), we obtain

$$
\tau_{k}=\frac{1}{\omega_{0}} \arccos \left(\frac{\left(b_{5} b_{1}-b_{4}\right) \omega_{0}^{4}+\left(b_{4} b_{2}-b_{3} b_{1}\right) \omega_{0}^{2}}{-b_{5}^{2} \omega_{0}^{4}+\left(2 b_{3} b_{5}-b_{4}^{2}\right) \omega_{0}^{2}-b_{3}^{2}}\right)+\frac{2 k \pi}{\omega_{0}} ; \quad k=0,1,2, \ldots
$$

Theorem 2 Assume that

$$
b_{1}+b_{5}>0, \quad b_{3}>0, \quad\left(b_{1}+b_{5}\right)\left(b_{2}+b_{4}\right)-b_{3}>0
$$

hold.

(i) If $\gamma \geq 0$ and $\beta>0$, then $E^{0}$ is locally asymptotically stable for any $\tau \geq 0$.

(ii) If either $\gamma<0$ or $\gamma \geq 0$ and $\beta<0$, then

- $E^{0}$ is locally asymptotically stable when $\tau<\tau_{0}$,

- $E^{0}$ undergoes a Hopf bifurcation when $\tau=\tau_{0}$,

- $E^{0}$ is unstable when $\tau>\tau_{0}$,

where

$$
\tau_{0}=\frac{1}{\omega_{0}} \arccos \left(\frac{\left(b_{5} b_{1}-b_{4}\right) \omega_{0}^{4}+\left(b_{4} b_{2}-b_{3} b_{1}\right) \omega_{0}^{2}}{-b_{5}^{2} \omega_{0}^{4}+\left(2 b_{3} b_{5}-b_{4}^{2}\right) \omega_{0}^{2}-b_{3}^{2}}\right)
$$

\section{$3.3 \tau \neq 0$ and $f(\tau)=e^{-k_{31} \tau}$}

Since there is still only a Gd-NPs-free equilibrium $\left(E^{0}\right)$ for this case, we follow a similar approach in the previous part to explore stability of the equilibrium. Hence, the charac- 
teristic equation of the linearized system is given by

$$
R(\lambda, \tau)=\lambda^{3}+b_{1} \lambda^{2}+b_{2} \lambda+\left(b_{3}+b_{4} \lambda+b_{5} \lambda^{2}\right) e^{-k_{31} \tau} \cdot e^{-\lambda \tau}=0
$$

with $b_{n}$ for $n=1, \ldots, 5$.

Substituting $\lambda(\tau)=i \omega(\tau)$ with $\omega>0$ into Eq. (7), we have

$$
\left(-i \omega^{3}-b_{1} \omega^{2}+i b_{2} \omega\right)+\left(b_{3}-b_{5} \omega^{2}+i b_{4} \omega\right)(\cos \omega \tau-i \sin \omega \tau) e^{-k_{31} \tau}=0
$$

By separating real and imaginary parts in Eq. (8), the real part satisfies the following equation:

$$
-b_{1} \omega^{2}+b_{3} \cos \omega \tau \cdot e^{-k_{31} \tau}+b_{4} \omega \sin \omega \tau \cdot e^{-k_{31} \tau}-b_{5} \omega^{2} \cos \omega \tau \cdot e^{-k_{31} \tau}=0,
$$

while the imaginary part satisfies the equation

$$
-\omega^{3}+b_{2} \omega-b_{3} \sin \omega \tau \cdot e^{-k_{31} \tau}+b_{4} \omega \cos \omega \tau \cdot e^{-k_{31} \tau}+b_{5} \omega^{2} \sin \omega \tau \cdot e^{-k_{31} \tau}=0 .
$$

Next, Eq. (10) can be rewritten as

$$
\sin \omega \tau \cdot e^{-k_{31} \tau}=\frac{\omega^{3}-b_{2} \omega-b_{4} \omega \cos \omega \tau \cdot e^{-k_{31} \tau}}{b_{5} \omega^{2}-b_{3}} .
$$

Then, substituting Eq. (11) into Eq. (9) yields

$$
\cos \omega \tau=\frac{\left(b_{1} b_{5}-b_{4}\right) \omega^{4}+\left(b_{2} b_{4}-b_{1} b_{3}\right) \omega^{2}}{-b_{5}^{2} \omega^{4}+\left(2 b_{3} b_{5}-b_{4}^{2}\right) \omega^{2}-b_{3}^{2}} \cdot e^{-k_{31} \tau}
$$

Notice that Eq. (12) is a transcendental equation and can be very difficult to solve for a closed-form solution. Hence, we use a geometrical approach by considering intersections of two curves from the equation. Let us define $f_{1}=\cos \omega \tau$ and $f_{2}=\frac{\left(b_{1} b_{5}-b_{4}\right) \omega^{4}+\left(b_{2} b_{4}-b_{1} b_{3}\right) \omega^{2}}{-b_{5}^{2} \omega^{4}+\left(2 b_{3} b_{5}-b_{4}^{2}\right) \omega^{2}-b_{3}^{2}}$. $e^{-k_{31} \tau}$. Based on parameter ranges of this study, there is no intersection between those two curves. Hence, we cannot find $\omega$ that satisfies Eq. (12) (see Fig. 2(a)-(d)). Consequently, there is no periodic solution of the system.

Theorem 3 If Eq. (12) has no $\omega$, then $E^{0}$ is always locally asymptotically stable for all $\tau>0$.

\section{Numerical results}

In this section, numerical results for dynamical behaviors of Gd-NPs in bloodstream, peripheral arteries, and vasa vasorum are illustrated to support theoretical results obtained from the previous section and to demonstrate the effects of transfer rates and the sojourn time that Gd-NPs remain trapped in the vasa vasorum compartment. Note that the numerical simulations are based on the Runge-Kutta method of order 4 incorporated with the initial conditions for solving delay differential equations [26].

In case $f(\tau)=1$ for $\tau>0$ or equivalently Gd-NPs are trapped in the vasa vasorum at a fixed length of time before escaping to other parts, dynamical behaviors of Gd-NPs according to different values of $\tau(0.3,1.7,1.96,2.5)$ are shown in Fig. 3. As a critical value of $\tau$ 

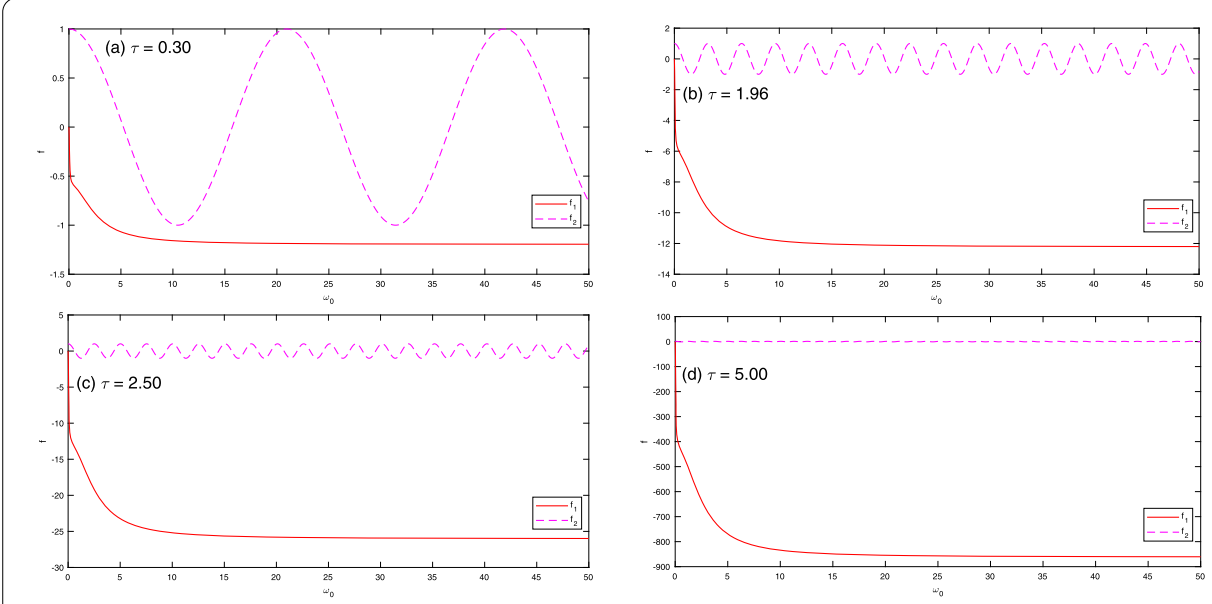

Figure 2 Based on the parameter values, there are no intersections between $f_{1}$ and $f_{2}$ so that there are no imaginary roots for the delay system with $f(\tau)=e^{-k_{31} \tau}$
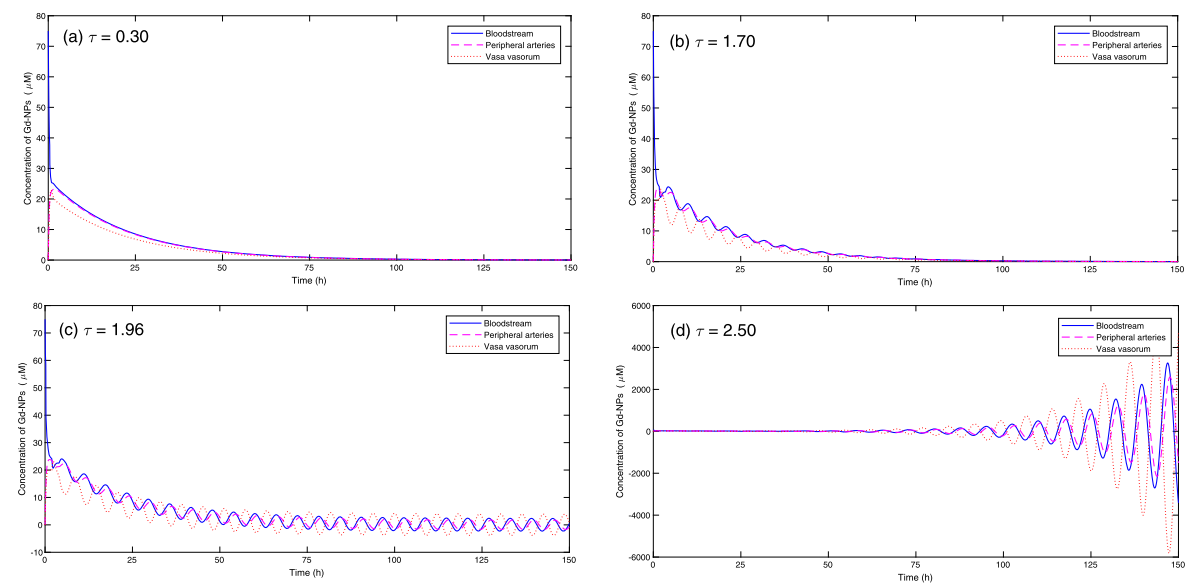

Figure 3 Dynamical behaviors of Gd-NPs according to $\tau$ for $f(\tau)=1$ (with $\tau_{0}=1.96$ and the initial condition $\left.\left(y_{1}(0), y_{2}(0), y_{3}(0)\right)=(75,0,0)\right)$. (a) When $\tau=0.30$, a solution of system (1) approaches the Gd-NPs-free equilibrium or Gd-NPs are eventually eliminated from every compartment. (b) When $\tau=1.70$, a solution oscillates and then eventually tends to the NPs-free equilibrium or the concentration of Gd-NPs fluctuates at a certain period at the beginning of time before Gd-NPs being washed out. (c) When $\tau=1.96$, a solution oscillates periodically or Gd-NPs periodically circulate in the system. (d) When $\tau=2.5$, a solution diverges from the Gd-NPs-free equilibrium or Gd-NPs behaviors are unstable

$\left(\tau_{0}\right)$ based on the parameter values in Table 1 is approximately 1.96 hours, Gd-NPs dynamics change around such a value. If $\tau<1.96$, the system solution tends to the Gd-NPs-free equilibrium, and it starts oscillating when $\tau$ becomes closer to $\tau_{0}$ (see Fig. 3(a)-(b)). The results correspond to Theorem 2 that the Gd-NPs-free equilibrium of system (1) is locally asymptotically stable when $\tau<1.96$. At $\tau=1.96$, Fig. 3(c) shows that the solution fluctuates in a periodic pattern. Those dynamical behaviors are in agreement with Theorem 2 so that a Hopf bifurcation occurs at $\tau=\tau_{0}=1.96$. In Fig. 3(d), when $\tau>1.96$, the solution fluctuates with an increasing amplitude and becomes divergent. This is in agreement with the unstable behaviors around the equilibrium after $\tau>\tau_{0}$ in Theorem 2. In Fig. 4, we demonstrate phase portraits at different values of $\tau$ in correspondence with results in 

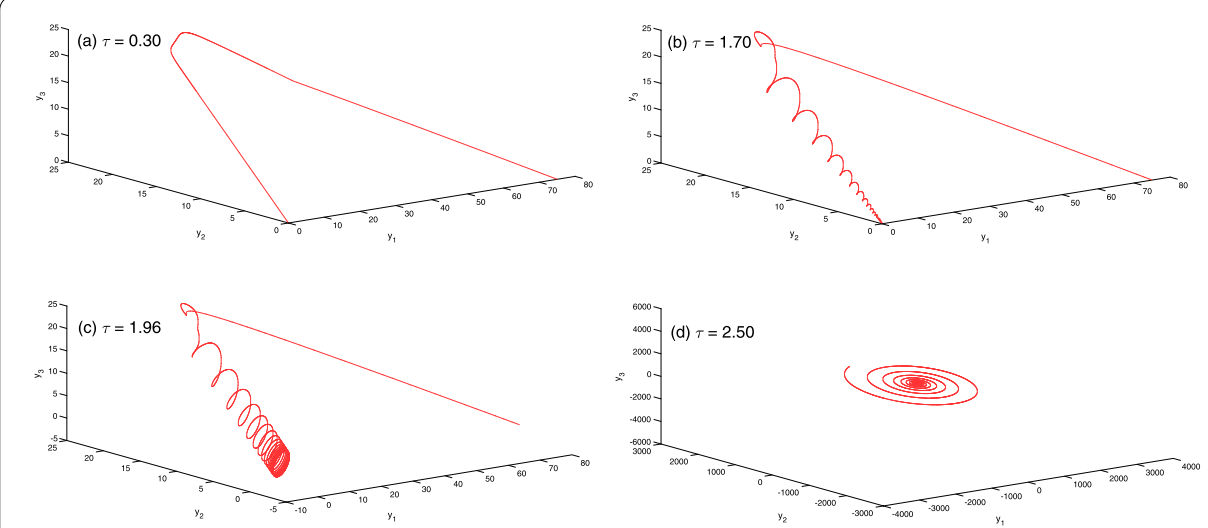

Figure 4 Phase portraits that show the dynamical behaviors of (1) near the Gd-NPs-free equilibrium at (a) $\tau=0.30$, (b) $\tau=1.70$, (c) $\tau=1.96$, and (d) $\tau=2.5$ for $f(\tau)=1$. The results demonstrate oscillation behaviors of the Gd-NPs concentration near the equilibrium when $\tau$ increases
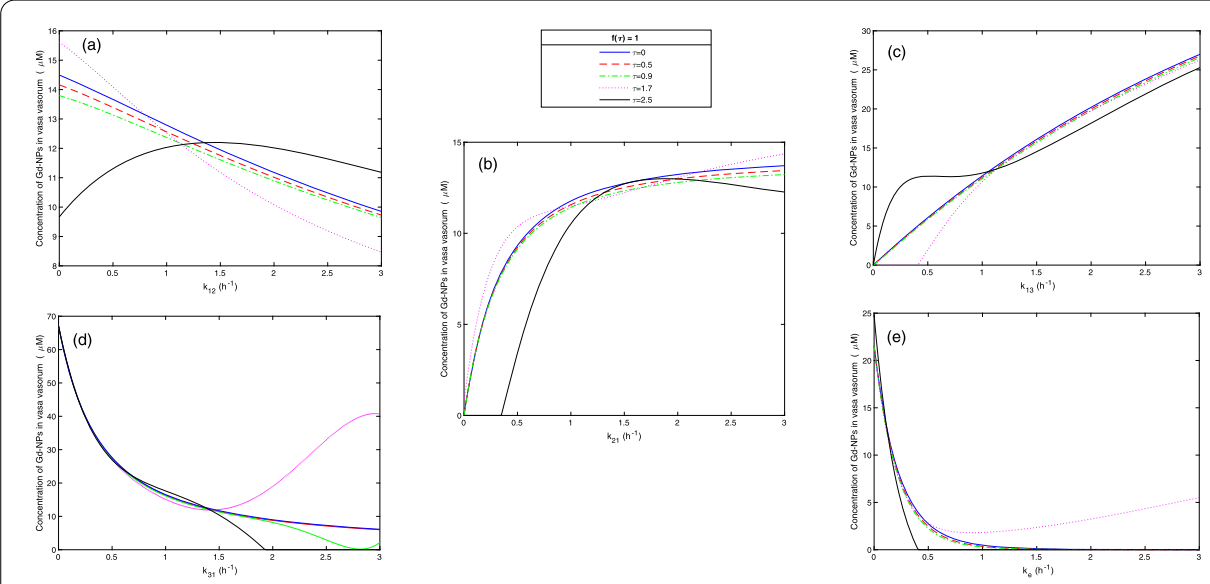

Figure 5 The concentration of Gd-NPs in vasa vasorum at the 12th hour after the initial injection for $f(\tau)=1$ when transfer rates and $\tau$ are varied

Fig. 3. In Fig. 4(a)-(b), with $\tau=0.3$ and $\tau=1.7$, solution trajectories approach the origin without and with fluctuation. The trajectory neither tends toward the origin nor infinity if $\tau=1.96$ in Fig. 4(c). When $\tau=2.5$, the solution trajectory diverges to infinity over time (see Fig. 4(d)).

Next, we investigate the effects of transfer rates on the concentration of Gd-NPs at the 12th hour after the initial injection according to different values of $\tau$ (see Fig. 5). Results in Fig. 5(a) suggest that increasing the transfer rate of Gd-NPs from bloodstream to peripheral arteries may result in the decreased concentration of Gd-NPs in vasa vasorum. However, such a trend of results may slightly be affected by the increasing value of $\tau$. In Fig. 5(b), the transfer rate from peripheral arteries to bloodstream has a significant impact on the concentration of Gd-NPs when it is quite small. When it becomes larger, the concentration gradually increases. The increasing value of $\tau$ may alter the trend by delaying the increasing concentration of Gd-NPs. Results in Fig. 5(c) demonstrate the increasing concentration of Gd-NPs according to the transfer rate from bloodstream to vasa vasorum. Figure 5(d) suggests that increasing the transfer rate from vasa vasorum to blood- 

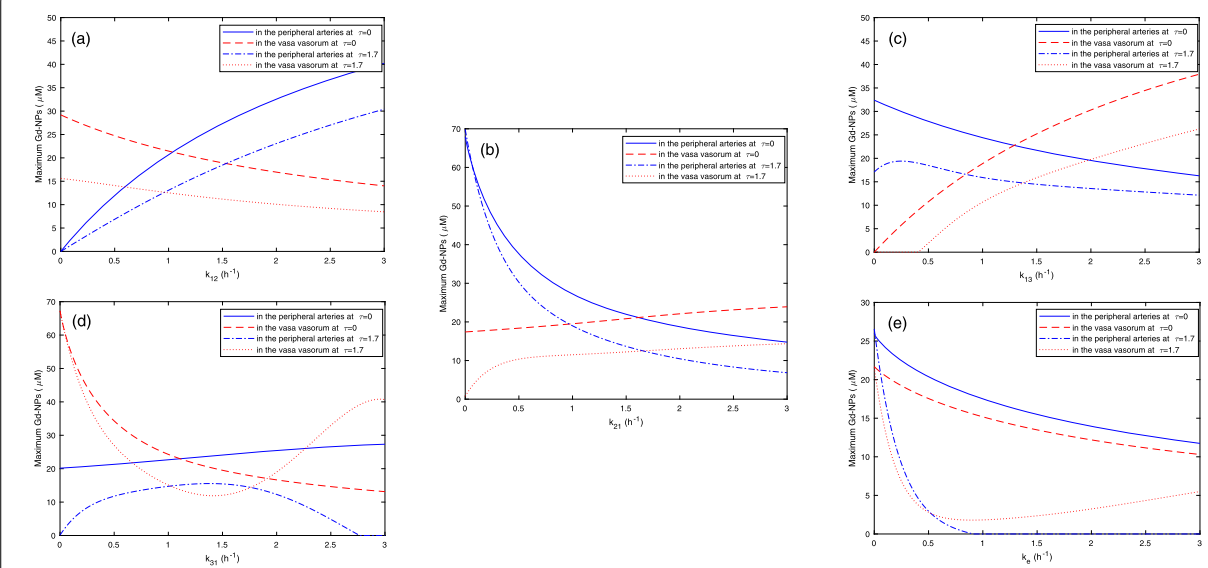

Figure 6 The maximum concentration of Gd-NPs in peripheral arteries and vasa vasorum without and with particles remaining trapped in vasa vasorum $(\tau=0,1.7)$ for $f(\tau)=1$

stream may result in the reduction of Gd-NPs in vasa vasorum. Similar to the previous results, the increasing value of $\tau$ may affect the result trend. In Fig. 5(e), the higher elimination rate drastically reduces the concentration of Gd-NPs in vasa vasorum and higher $\tau$ may affect the normal trend of results.

Moreover, we explore the effects of transfer rates on the maximum concentration of Gd-NPs inside peripheral arteries and vasa vasorum when Gd-NPs do not and do remain in vasa vasorum at the fixed length of time $\tau=0,1.7$. Note that based on the preceding study from the preceding work, Gd-NPs remain trapped in vasa vasorum for approximately $\tau=1.7$ hours. In Fig. 6, the overall results suggest similar trends of results according to transfer rates for both $\tau=0$ and $\tau=1.7$, but the concentration of Gd-NPs for the $\tau=0$ case is generally higher. Our results in Fig. 6(a) demonstrate that when the transfer rate from bloodstream to peripheral arteries increases, the concentration of Gd-NPs in peripheral arteries considerably increases, while the concentration of Gd-NPs in vasa vasorum moderately decreases. Figure 6(b) shows the significantly decreasing concentration of Gd-NPs in peripheral arteries and the slightly increasing concentration of Gd-NPs in vasa vasorum according to the increasing transfer rate from peripheral arteries to bloodstream. In Fig. 6(c), when the transfer rate from bloodstream to vasa vasorum increases, it may result in the increase of Gd-NPs concentration in vasa vasorum but in the moderate decrease of Gd-NPs concentration in peripheral arteries. The increasing transfer rate from vasa vasorum to bloodstream may lead to the decreasing concentration of Gd-NPs in vasa vasorum but the slightly increasing concentration of Gd-NPs in peripheral arteries for $\tau=0$ and the slightly increasing then decreasing concentration of Gd-NPs in peripheral arteries for $\tau=1.7$ (see Fig. 6(d)). In addition, when the sojourn time is present, it may also result in the increasing concentration after the reduction of Gd-NPs in vasa vasorum. Our results in Fig. 6(e) demonstrate that the concentration of Gd-NPs in vasa vasorum and peripheral arteries decreases if the elimination rate increases.

In the case $f(\tau)=e^{-k_{31} \tau}$ for $\tau \neq 0$ or equivalently Gd-NPs are trapped in the vasa vasorum at a fixed length of time and eliminated during the stay at a rate of $e^{-k_{31} \tau}$ before escaping to other parts, time-series plots and phase portraits that represent the dynamical behaviors near the Gd-NPs-free equilibrium for $\tau=0.3$ and $\tau=2.5$ are illustrated in 

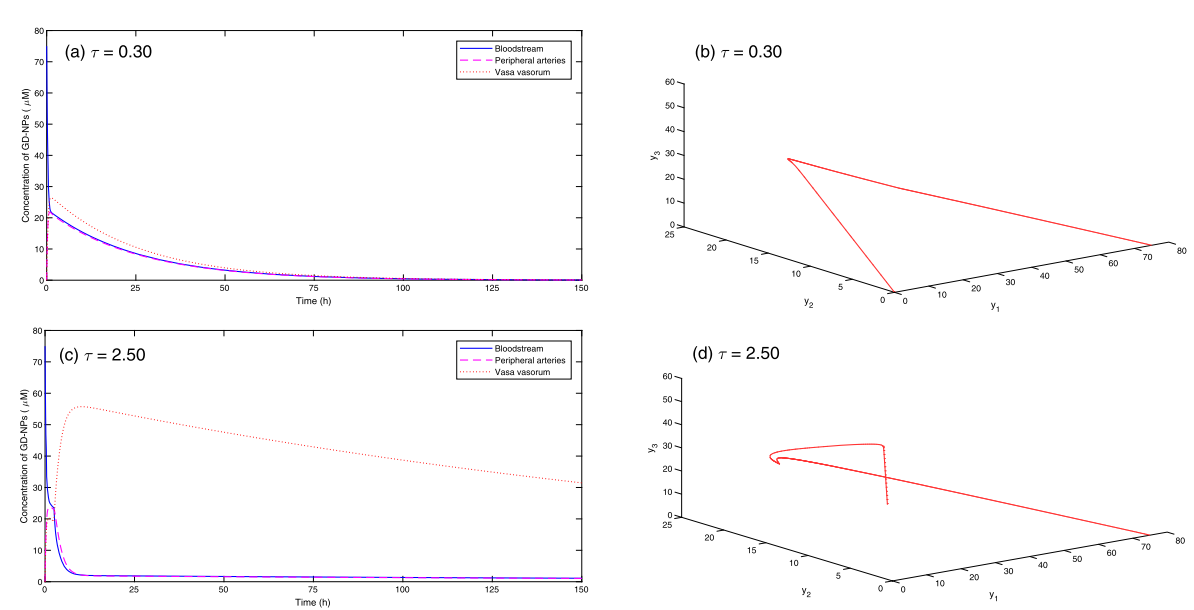

Figure 7 Dynamical behaviors of Gd-NPs according to $\tau$ for $f(\tau)=e^{-k_{31} \tau}$. (a) The concentration of Gd-NPs changes according to time when $\tau=0.30$. (b) A phase portrait of (1) when $\tau=0.3$. It shows that a solution approaches the Gd-NPs-free equilibrium (c) The concentration of Gd-NPs changes according to time when $\tau=2.5$. (d) A phase portrait of (1) when $\tau=2$.5. Eventually, a solution will approach the Gd-NPs-free equilibrium

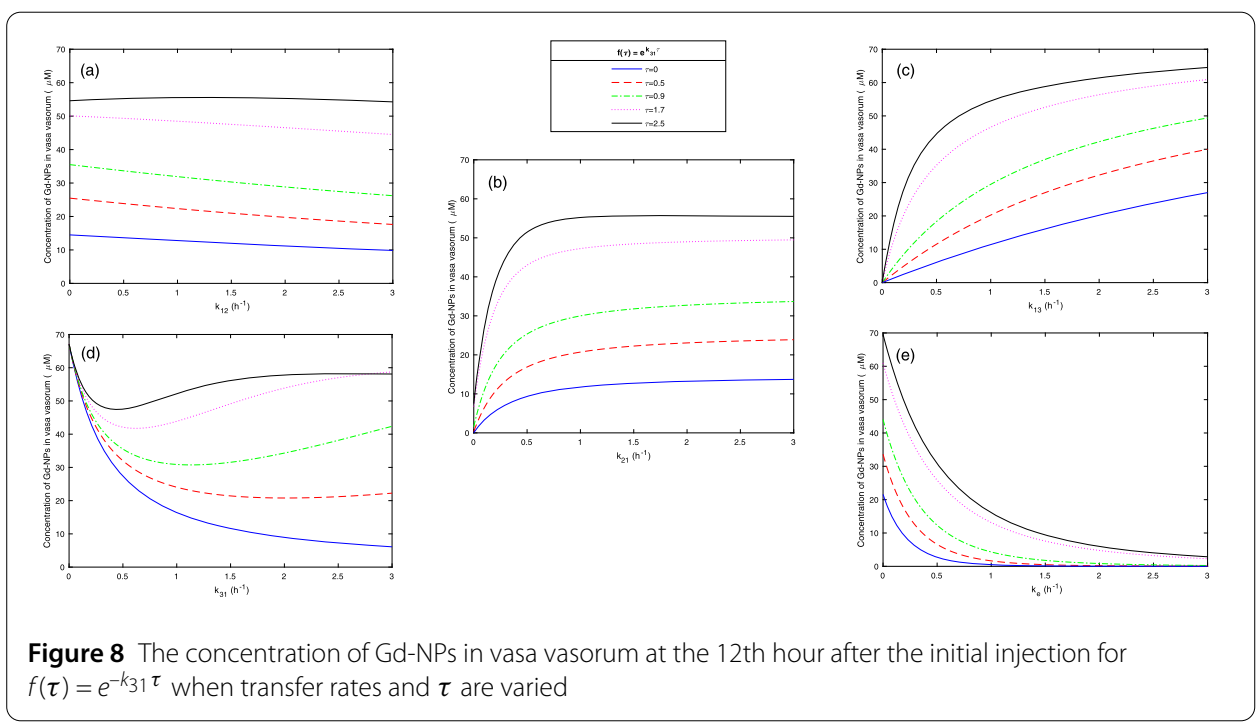

Fig. 7. Figure 7(a)-(b) demonstrate the concentration of Gd-NPs according to time that Gd-NPs are eventually eliminated and a phase portrait that shows a solution approaching the origin for $\tau=0.3$. In Fig. 7(c)-(d), our results show that the concentration of Gd-NPs approaches zero according to time and a corresponding phase portrait that shows a solution approaching the origin for $\tau=2.5$.

We further investigate the effects of transfer rates on the concentration of Gd-NPs at the 12th hour after the initial injection when $f(\tau)=e^{-k_{31} \tau}$ for $\tau \neq 0$. Overall, our results suggest that increasing $\tau$ leads to the higher concentration of Gd-NPs. In Fig. 8(a), the transfer rate of Gd-NPs from bloodstream to peripheral arteries rarely affects the concentration of Gd-NPs. Figure 8(b) shows that when the transfer rate from peripheral arteries to bloodstream increases, the concentration of Gd-NPs increases at the beginning before becoming saturated according to the transfer rate. The increasing transfer rate of Gd-NPs 

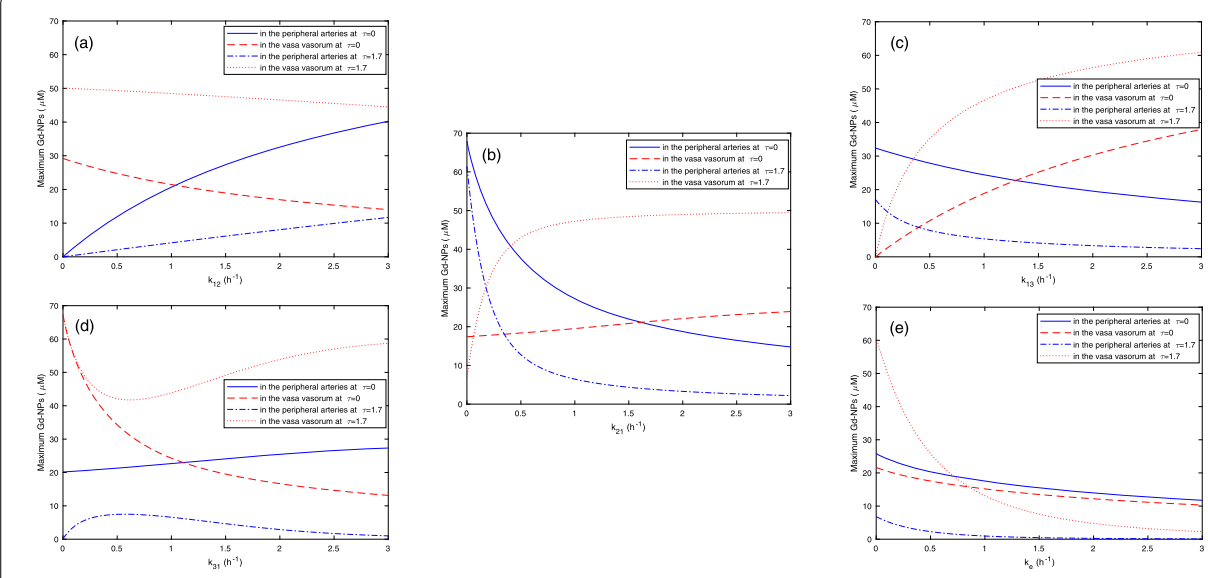

Figure 9 The maximum concentration of Gd-NPs in peripheral arteries and vasa vasorum without and with particles remaining trapped in vasa vasorum $(\tau=0,1.7)$ for $f(\tau)=e^{-k_{31} \tau}$

from bloodstream to vasa vasorum significantly influences the concentration of Gd-NPs and results in higher concentration (see Fig. 8(c)). In contrast, when the transfer rate from vasa vasorum to bloodstream increases, the concentration of Gd-NPs significantly decreases as shown in Fig. 8(d). Figure 8(e) illustrates that the increasing clearance rate may reduce the concentration of Gd-NPs in vasa vasorum.

Figure 9(a)-(e) illustrates that the longer sojourn time that Gd-NPs remain trapped generally results in the higher concentration of Gd-NPs in vasa vasorum and the lower concentration of Gd-NPs in peripheral arteries. In Fig. 9(a), increasing the transfer rate of Gd-NPs from bloodstream to peripheral arteries may moderately increase the concentration of Gd-NPs in peripheral arteries and slightly reduce the concentration of Gd-NPs in vasa vasorum. Results in Fig. 9(b) suggest that increasing the transfer rate of Gd-NPs from peripheral arteries to bloodstream may result in the significant decrease of Gd-NPs concentration in peripheral arteries and the small increase of Gd-NPs concentration in vasa vasorum. As illustrated in Fig. 9(c), when the transfer rate of Gd-NPs from bloodstream to vasa vasorum increases, the concentration of Gd-NPs in peripheral arteries intermediately decreases, while the concentration of Gd-NPs in vasa vasorum considerably increases. In Fig. 9(d), increasing the transfer rate of Gd-NPs from vasa vasorum to bloodstream may result in the slightly decreasing concentration of Gd-NPs in peripheral arteries and the significantly increasing concentration of Gd-NPs in vasa vasorum when the sojourn of Gd-NPs in vasa vasorum is present. The increasing elimination rate of Gd-NPs generally leads to the decreasing concentration of Gd-NPs in both peripheral arteries and vasa vasorum (see Fig. 9(e)). When results in Figs. 6 and 9 are compared, the difference of $f(\tau)$ may affect the concentration trends in vasa vasorum. When Gd-NPs remain trapped in vasa vasorum, the concentration of Gd-NPs is lower than when they do not for $f(\tau)=1$, while it is higher for $f(\tau)=e^{-k_{31} \tau}$.

\section{Conclusion}

In this study, the three-compartmental pharmacokinetic model for describing the distribution of Gd-NPs used in the contrast enhanced MRI is extended to incorporate a sojourn period of particle circulation in vasa vasorum. The particle dynamics are then described 
by a system of delay differential equations. Two types of circulation are considered: with and without natural clearance during the particle sojourn.

When the sojourn of Gd-NPs in vasa vasorum is not included $(\tau=0)$, we show that the particle-free equilibrium point is locally asymptotically stable. When the fixed period of circulation is present $(\tau \neq 0)$, the delay system is analyzed to determine the conditions for asymptomatic stability and the critical time of a periodic solution. Our findings suggest that only when Gd-NPs are not eliminated during the sojourn in vasa vasorum, a periodic solution occurs under certain conditions. If Gd-NPs are eliminated by natural clearance during their stay in vasa vasorum, a system solution always tends to the particle-free equilibrium point.

In addition, we demonstrate the dynamics of Gd-NPs numerically according to the stability conditions. Our results are in agreement with the conditions and show asymptotic, periodic, and unstable solution behaviors of Gd-NPs when there is no clearance of GdNPs during their stay in vasa vasorum $(f(\tau)=1)$. Generally, a solution starts to oscillate when $\tau$ approaches its critical value $(\tau=1.96)$, shows a periodic behavior when $\tau=1.96$, and then shows an unstable behavior when $\tau>1.96$. Consequently, to ensure that GdNPs are completely eliminated, Gd-NPs should be designed not to linger in vasa vasorum for too long. However, if Gd-NPs are naturally cleared during their stay in vasa vasorum $\left(f(\tau)=e^{-k_{31} \tau}\right)$, the particle-free equilibrium point is asymptotically stable. In such a case, a solution will tend to the equilibrium point or Gd-NPs will always be eventually eliminated from the body. As $\tau$ is normally less than the critical value in reality, we further investigate how transfer rates of Gd-NPs affect their presence in blood stream, peripheral arteries, and vasa vasorum after twelve hours of injection, and also the maximum concentration of Gd-NPs. It is found that increasing transfer rates $\left(k_{12}, k_{31}\right.$, and $\left.k_{e}\right)$ lowers the concentration of Gd-NPs, while increasing $k_{21}$ and $k_{13}$ leads to the higher concentration of Gd-NPs. For the maximum concentration, our results suggest that transfer rates affect the concentration of Gd-NPs in peripheral arteries and vasa vasorum. Note that results are similar for both types of Gd-NPs circulation. In addition, overall results suggest that increasing $\tau$ may result in the higher concentration of Gd-NPs. Moreover, the maximum of Gd-NPs in vasa vasorum for $f(\tau)=1$ is generally lower than that for $f(\tau)=e^{-k_{31} \tau}$.

Finally, we believe that this study may help gain a better understanding into the effects of the fixed period of particle circulation and transfer parameters, may also help design a therapy that enhances the efficacy of MRI, and may suggest possible administration strategies that could reduce toxicity from overdose.

Acknowledgements

AP and FC would like to thank Assoc. Prof. Charin Modchang and Asst. Prof. Pairote Satiracoo for suggestive comments.

\section{Funding}

AP was partially financially supported by the Centre of Excellence in Mathematics, the Commission on Higher Education, Thailand for her tuition fees. The funding body had no role in the study design, analysis, and interpretation of results nor in writing the manuscript.

\footnotetext{
Abbreviations

Gd-NPs, Gadolinium nanoparticles; MRI, Magnetic resonance imaging; NPs, Nanoparticles; GBCAs, Gadolinium-based contrast agents; PBPK, Physiologically-based pharmacokinetic; tf, Transferrin; siRNA, Small interfering RNA; CTCS, Concentration-time curves; Gd-CTCs, Gadolinium contrast agent concentration-time curves; PAA, Polyacrylamide; PAApeg, Polyethylene glycol-coated polycrylamide; Gd-DTPA, Gadolinium-diethylenetriamine pentaacetic acid; AUC, Area under the curve; DCE-MRI, Dynamic contrast enhanced-magnetic resonance imaging.
} 


\section{Competing interests}

The authors declare that they have no competing interests.

\section{Authors' contributions}

All authors contributed equally to this work. All authors read and approved the final manuscript.

\section{Publisher's Note}

Springer Nature remains neutral with regard to jurisdictional claims in published maps and institutional affiliations.

Received: 31 July 2020 Accepted: 4 November 2020 Published online: 25 November 2020

\section{References}

1. Gisterå, A., Hansson, G.: The immunology of atherosclerosis. Nat. Rev. Nephrol. 13, 368-380 (2017). https://doi.org/10.1038/nrneph.2017.51

2. Degnan, A.J., Patterson, A.J., Tang, T.Y., Howarth, S.P.S., Gillard, J.H.: Evaluation of ultrasmall superparamagnetic iron oxide-enhanced MRI of carotid atherosclerosis to assess risk of cerebrovascular and cardiovascular events: follow-up of the ATHEROMA trial. Cerebrovasc. Dis. 34, 169-173 (2012). https://doi.org/10.1159/000339984

3. Kurt, E.J. (ed.): Histology and Cell Biology Lippincott Williams and Wilkins (1991)

4. Staub, D., Schinkel, A.F., Coll, B., Coli, S., van der Steen, A.F., Reed, J.D., Krueger, C., Thomenius, K.E., Adam, D., Sijbrands, E.J., ten Cate, F.J., Feinstein, S.B.: Contrast-enhanced ultrasound imaging of the vasa vasorum: from early atherosclerosis to the identification of unstable plaques. JACC Cardiovasc. Imaging. 3(7), 761-771 (2010). https://doi.org/10.1016/j.jcmg.2010.02.007

5. Kubo, T., Akasaka, T.: Gray-scale intravascular ultrasound sheds light on the importance of vasa vasorum in unstable coronary plaque. J. Cardiol. 69(4), 599-600 (2017). https://doi.org/10.1016/j.jjcc.2016.11.001

6. Winter, P.M., Neubauer, A.M., Caruthers, S.D., Harris, T.D., Robertson, J.D., Williams, T.A., Schmieder, A.H., Hu, G., Allen, J.S., Lacy, E.K., Zhang, H., Wickline, S.A., Lanza, G.M.: Endothelial alpha(v)beta3 integrin-targeted fumagillin nanoparticles inhibit angiogenesis in atherosclerosis. Arterioscler. Thromb. Vasc. Biol. 26(9), 2103-2109 (2006). https://doi.org/10.1161/01.ATV.0000235724.11299.76

7. Ouimet, T., Lancelot, E., Hyafil, F., Rienzo, M., Deux, F., Lemaître, M., Duquesnoy, S., Garot, J., Roques, B.P., Michel, J.B., Corot, C., Ballet, S.: Molecular and cellular targets of the MRI contrast agent P947 for atherosclerosis imaging. Mol. Pharm. 9(4), 850-861 (2012). https://doi.org/10.1021/mp2003863

8. Calcagno, C., Ramachandran, S., Millon, A., Robson, P.M., Mani, V., Fayad, Z.: Gadolinium-based contrast agents for vessel wall magnetic resonance imaging (MRI) of atherosclerosis. Curr. Cardiovasc. Imaging Rep. 6(1), 11-24 (2013). https://doi.org/10.1007/s12410-012-9177-x

9. Zhou, Z., Qutaish, M., Han, Z., Schur, R.M., Liu, Y., Wilson, D.L., Lu, Z.R.: MRI detection of breast cancer micrometastases with a fibronectin-targeting contrast agent. Nat. Commun. 6, 7984 (2015). https://doi.org/10.1038/ncomms8984

10. Gale, M.E., Caravan, P.: Gadolinium-free contrast agents for magnetic resonance imaging of the central nervous system. ACS Chem. Neurosci. 9(3), 395-397 (2018). https://doi.org/10.1021/acschemneuro.8b00044

11. Xiao, Y., Paudel, R., Liu, J., Ma, C., Zhang, Z., Zhou, S.: MRI contrast agents: classification and application (review). Int. J. Mol. Med. 38, 1319-1326 (2016). https://doi.org/10.3892/ijmm.2016.2744

12. Ruiz, A., Hernández, Y., Cabal, C., González, E., Veintemillas-Verdaguer, S., Martínez, E., Morales, M.P.: Biodistribution and pharmacokinetics of uniform magnetite nanoparticles chemically modified with polyethylene glycol. Nanoscale 5 , 11400-11408 (2013). https://doi.org/10.1039/C3NR01412F

13. Iwaki, S., Hokamura, K., Ogawa, M., Takehara, Y., Muramatsu, Y., Yamane, T., Hirabayashi, K., Morimoto, Y., Hagisawa, K., Nakahara, K., Mineno, T., Terai, T., Komatsu, T., Ueno, T., Tamura, K., Adachi, Y., Hirata, Y., Arita, M., Arai, H., Umemura, K., Naganok, T., Hanaoka, K.: A design strategy for small molecule-based targeted MRI contrast agents: their application for detection of atherosclerotic plaques. Org. Biomol. Chem. 12, 8611-8618 (2014). https://doi.org/10.1039/c4ob01270d

14. Woodside, D.G., Tanifum, E.A., Ghaghada, K.B., Biediger, R.J., Caivano, A.R., Starosolski, Z.A., Khounlo, S., Bhayana, S., Abbasi, S., Craft, J.W., Maxwell, D.S., Patel, C., Stupin, I.V., Bakthavatsalam, D., Market, R.V., Willerson, J.T., Dixon, R.A.F., Vanderslice, P., Annapragada, A.V.: Magnetic resonance imaging of atherosclerotic plaque at clinically relevant field strengths (1T) by targeting the integrin $\alpha 4 \beta$ 1. Sci. Rep. 8, 3733 (2018). https://doi.org/10.1038/s41598-018-21893-x

15. Burtea, C., Laurent, S., Murariu, O., Rattat, D., Toubeau, G., Verbruggen, A., Vansthertem, D., Elst, L.V., Muller, R.N.: Molecular imaging of $\alpha_{v} \beta_{3}$ integrin expression in atherosclerotic plaques with a mimetic of RGD peptide grafted to Gd-DTPA. Cardiovasc. Res. 78(1), 148-157 (2008). https://doi.org/10.1093/cvr/cvm115

16. Bartlett, D.W., Su, H., Hildebrandt, I.J., Weber, W.A., Davis, M.E.: Impact of tumor-specific targeting on the biodistribution and efficacy of siRNA nanoparticles measured by multimodality in vivo imaging. Proc. Natl. Acad. Sci. USA 104(39), 15549-15554 (2007). https://doi.org/10.1073/pnas.0707461104

17. Barboriak, D.P., MacFall, J.R., Viglianti, B.L., Dewhirst, M.W.: Comparison of three physiologically-based pharmacokinetic models for the prediction of contrast agent distribution measured by dynamic MR imaging. J. Magn. Reson. Imaging 27, 1388-1398 (2008). https://doi.org/10.1002/jmri.21344

18. Neubauer, A.M., Sim, H., Winter, P.M., Caruthers, S.D., Williams, T.A., Robertson, J.D., Sept, D., Lanza, G.M., Wickline, S.A.: Nanoparticle pharmacokinetic profiling in vivo using magnetic resonance imaging. Magn. Reson. Med. 60(6), 1353-1361 (2008). https://doi.org/10.1002/mrm.21795

19. Wenger, Y., Schneider, R.J., Reddy, G.R., Kopelman, R., Jolliet, O., Philbert, M.A.: Tissue distribution and pharmacokinetics of stable polyacrylamide nanoparticles following intravenous injection in the rat. Toxicol. Appl. Pharmacol. 251(3), 181-190 (2011). https://doi.org/10.1016/j.taap.2010.11.017

20. Taheri, S., Shah, N.J., Rosenberg, G.A.: Analysis of pharmacokinetics of Gd-DTPA for dynamic contrast-enhanced magnetic resonance imaging. Magn. Reson. Imaging 34(7), 1034-1040 (2016). https://doi.org/10.1016/j.mri.2016.04.014 
21. Li, T., Pintus, N., Viglialoro, G.: Properties of solutions to porous medium problems with different sources and boundary conditions. Z. Angew. Math. Phys. 70, Art. 86, 1-18 (2019). https://doi.org/10.1007/s00033-019-1130-2

22. Viglialoro, G., Woolley, T.E.: Boundedness in a parabolic-elliptic chemotaxis system with nonlinear diffusion and sensitivity and logistic source. Math. Methods Appl. Sci. 41, 1809-1824 (2018). https://doi.org/10.1002/mma.4707

23. Chiu, K.-S., Li, T.: Oscillatory and periodic solutions of differential equations with piecewise constant generalized mixed arguments. Math. Nachr. 292, 2153-2164 (2019). https://doi.org/10.1002/mana.201800053

24. Li, T., Rogovchenko, Y.V.: On the asymptotic behavior of solutions to a class of third-order nonlinear neutral differential equations. Appl. Math. Lett. 105, 1-7 (2020). https://doi.org/10.1016/j.aml.2020.106293

25. Bourafa, S., Abdelouahab, M.-S., Moussaoui, A.: On some extended Routh-Hurwitz conditions for fractional-order autonomous systems of order $\alpha \in(0,2)$ and their applications to some population dynamic models. Chaos Solitons Fractals 133, 109623 (2020). https://doi.org/10.1016/j.chaos.2020.109623

26. Baker, C.T.H., Paul, C.A.H., Willé, D.R.: Issues in the numerical solution of evolutionary delay differential equations. Adv. Comput. Math. 3, 171-196 (1995). https://doi.org/10.1007/BF02988625

Submit your manuscript to a SpringerOpen ${ }^{\circ}$ journal and benefit from:

- Convenient online submission

$\checkmark$ Rigorous peer review

Open access: articles freely available online

High visibility within the field

Retaining the copyright to your article

Submit your next manuscript at $\boldsymbol{\nabla}$ springeropen.com 